\title{
Pancreatitis and Biliary Obstruction Secondary to Duodenal Metastasis from Rapidly Progressing Lung Adenocarcinoma Treated with Common Bile Duct Stenting
}

\author{
Robert Sean O'Neill ${ }^{a} \quad T^{\prime}$ Tuan Duong ${ }^{b}$ Welan Dionelab Claudia Rogge ${ }^{b, c}$ \\ Daniel Brungs ${ }^{a, c}$ \\ aDepartment of Oncology, The Wollongong Hospital, Wollongong, NSW, Australia; \\ bepartment of Gastroenterology, The Wollongong Hospital, Wollongong, NSW, Australia; \\ CIllawarra Health and Medical Research Institute, University of Wollongong, Wollongong, \\ NSW, Australia
}

\section{Keywords}

Lung neoplasm $\cdot$ Malignancy $\cdot$ Duodenum $\cdot$ Imaging, Pathology

\begin{abstract}
Non-small cell lung cancer (NSCLC) is characterised by diffuse metastases, with common sites being the brain, liver, bones, and adrenal glands. Small bowel metastasis from NSCLC is a rare phenomenon, particularly in patients with an adenocarcinoma histology. We report the case of a 56-year-old lung adenocarcinoma patient with a duodenal metastasis diagnosed on FDG/ PET-CT and confirmed on duodenal biopsy. Although initially asymptomatic, he subsequently presented with obstructive jaundice secondary to rapid local disease progression at the duodenal metastasis, requiring endoscopic intervention for biliary drainage. He was commenced on single agent pembrolizumab, with disease response on subsequent follow-up. This case highlights a rare case of gastrointestinal metastasis from NSCLC requiring endoscopic intervention due to rapid progression of the disease at the site of metastasis.
\end{abstract}

\section{Introduction}

Small bowel metastases originating from primary pulmonary carcinomas are uncommon. In contrast to other metastatic sites, such as bone and brain, small bowel metastases are often asymptomatic, and the true incidence may be underreported. Small bowel metastasis typically occurs in final stages of widespread disease and is associated with a dismal 
prognosis, with a median survival time of 3 months seen in an autopsy study [1]. When symptomatic, small bowel metastases usually present with abdominal pain, nausea, or signs of anaemia, and in severe cases, with small bowel obstruction or perforation, or obstructive jaundice secondary to extraluminal compression of the biliary tree [2-4].

We report the case of a 56-year-old male diagnosed with non-small cell lung cancer (NSCLC) presenting with symptomatic brain metastases who was found to have an asymptomatic duodenal metastasis. He developed obstructive jaundice requiring endoscopic retrograde cholangiopancreatography (ERCP) and the insertion of a common bile duct (CBD) metal stent for symptomatic relief.

\section{Case Report}

A 56-year-old male presented to the emergency department of a regional hospital with a 5 -day history of headache and dysarthria. On examination, mild asymmetry of his lower face was appreciable, with the remainder of the physical examination being unremarkable apart from a large ventral hernia palpable on abdominal examination. He had a past medical history of type 2 diabetes mellitus, hypertension, hypercholesterolaemia, chronic obstructive pulmonary disease, gastro-oesophageal reflux disease, past hepatitis C infection, and depression. His surgical history was significant with a laparoscopic cholecystectomy 7 years prior, complicated by a bile leak and intra-abdominal sepsis requiring laparotomy and washout, and a subsequent large ventral hernia. He had a significant smoking history of approximately 50 years along with intermittent cannabis use, and a previous history of intravenous drug use 20 years prior to presentation.

A non-contrast brain computed tomography (CT) in the emergency department demonstrated a large area of low density in the right frontoparietal region, with subsequent brain MRI revealing a rounded subcortical enhancing lesion in the right precentral gyrus with surrounding vasogenic oedema.

A CT of the chest, abdomen and pelvis demonstrated a 6.5 -cm mass in the left upper zone medially with streaky surrounding inflammatory changes in the thorax, along with several enlarged mediastinal and periportal lymph nodes. Staging FDG PET/CT revealed an area of photopenia involving the right cerebral cortex in association with a hypodensity on the low-dose CT scan due to the known brain metastasis. He had an intensely avid (SUV $\max 10.3$ ) large mass in the left upper lobe, with heterogenous distribution of tracer with a central area of photopenia suggestive of central necrosis, as indicated by the green arrow in Figure 1. There were multiple FDG-avid mediastinal, supraclavicular, and peri-portal lymph nodes, as well as an FDG-avid left adrenal mass. An intense area of FDG avidity in the distal duodenal (D2) region $\left(\mathrm{SUV}_{\max } 11.8\right)$ was identified as indicated by the blue arrow in Figure 1. The patient had no symptoms attributable to the duodenal mass at the time of the FDG PET/CT scan.

The patient was commenced on regular dexamethasone $4 \mathrm{mg}$ and a proton pump inhibitor and subsequently underwent a right frontal lobe biopsy under stereotactic guidance. Histopathology of the brain metastasis demonstrated a metastatic tumour with solid sheets of malignant cells with atypical nuclei, eosinophilic cytoplasm, and frequent mitosis. Immunohistochemistry showed the tumour cells were positive for CK7 and TTF-1, consistent with a metastatic adenocarcinoma from a primary lung tumour. There was no targetable mutation detected in exon 19-21 of the epithelial growth factor receptor (EGFR) gene, and ALK, ROS-1, and BRAF immunohistochemistry were negative. Expression of the programmed cell death ligand-1 (PD-L1) was high (>95\%), suggesting sensitivity to immunotherapy with checkpoint inhibitors.

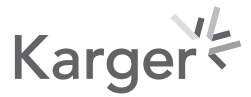


Fig. 1. FDG PET/CT demonstrating a large intensely FDG-avid mass in the left upper lobe medially and in the duodenum.

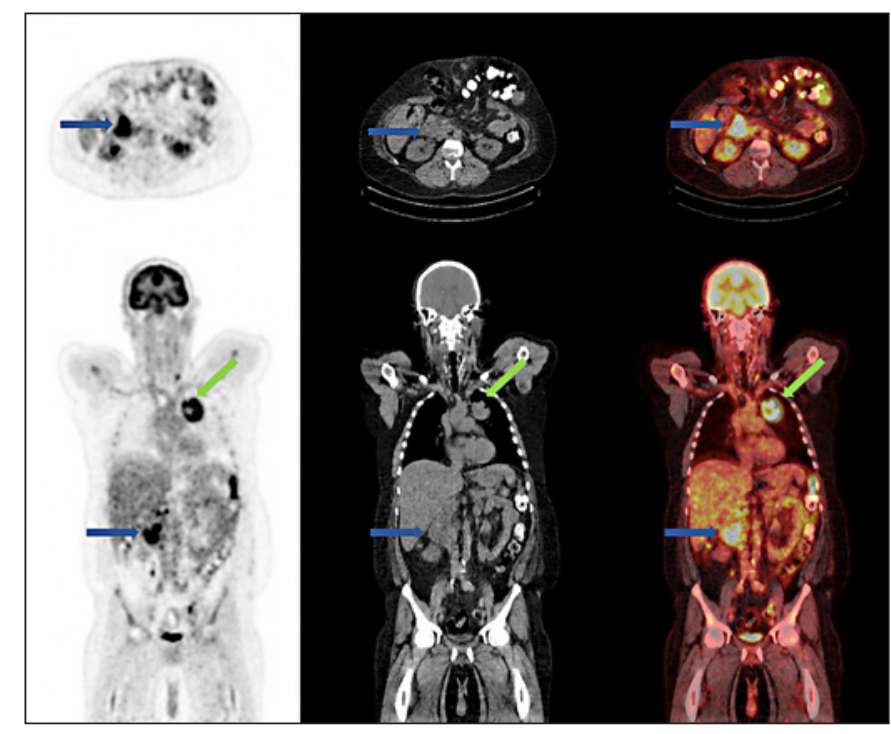

Fig. 2. Gastroscopy revealing a large semi-circumferential stenosing lesion in the second part of the duodenum and uncinate process.

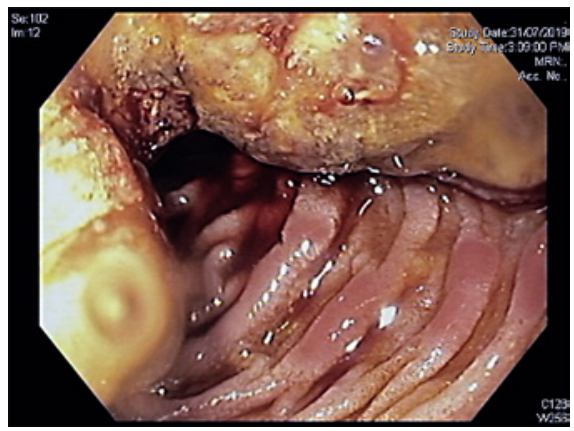

The patient underwent gastroscopy to investigate the FDG-avid duodenal lesion, which demonstrated a large semi-circumferential stenosing lesion in the second part of the duodenum, with ampullary orifice not visualised (Fig. 2). Endoscopic ultrasound showed significant peri-portal and peri-pancreatic lymphadenopathy, and a suspicious left adrenal gland nodule, confirming the finding of the PET scan. Biopsy of the duodenal mass demonstrated a TTF-1-positive adenocarcinoma consistent with metastatic lung adenocarcinoma and concordant with the resected brain metastasis.

The patient was discharged with the intention for treatment with post-operative cranial radiation and immunotherapy with pembrolizumab. He subsequently presented to the emergency department 12 days post initial discharge with a 4-day history of abdominal pain, dark urine, nausea, vomiting, and was jaundiced on clinical assessment. His liver function tests had become deranged in a predominately cholestatic pattern. Bilirubin was $74 \mu \mathrm{mol} / \mathrm{L}$, lipase was $654 \mathrm{U} / \mathrm{L}$, alkaline phosphatase (ALP) $427 \mathrm{U} / \mathrm{L}$, gamma-glutamyl transferase (GGT) $671 \mathrm{U} / \mathrm{L}$, alanine aminotransferase (ALT) $279 \mathrm{U} / \mathrm{L}$, and aspartate aminotransferase (AST) $108 \mathrm{U} / \mathrm{L}$. Abdominal CT demonstrated low-grade biliary dilatation due to the rapid increase in the size of the duodenal lesion. Inpatient duodenoscopy revealed a large fungating mass involving the ampulla of the duodenum with the bulk of the mass at the biliary orifice (Fig. 3a), and ERCP demonstrated extrinsic compression of the CBD with extrahepatic bile duct dilatation with tapering to the level of the ampullary tumour involvement, requiring the insertion of a distal CBD fully uncovered metal stent (Fig. 3b, c). 

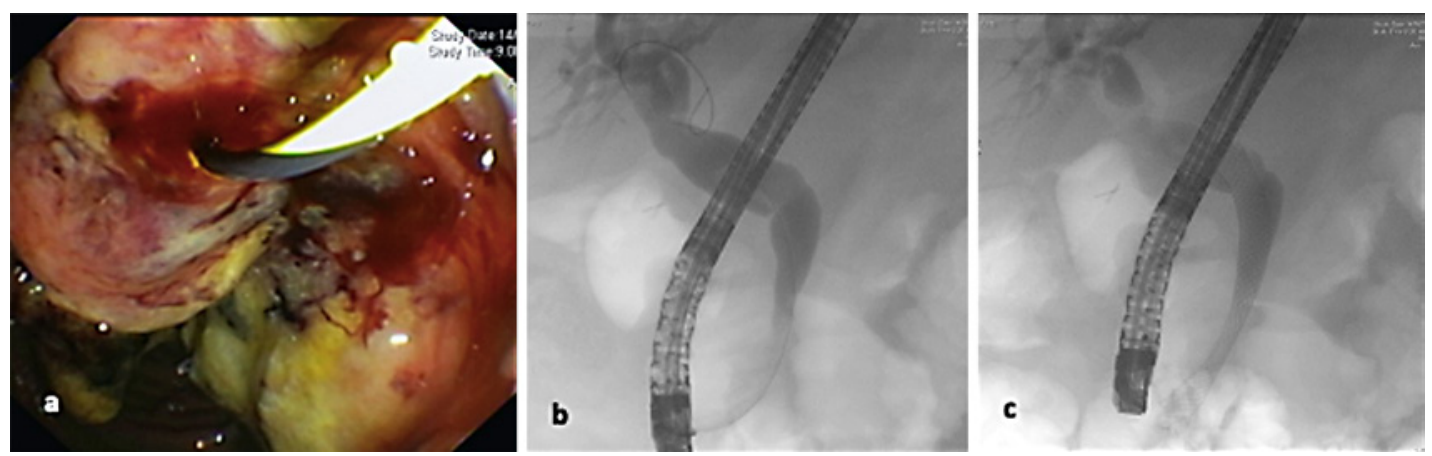

Fig. 3. a Duodenoscopy demonstrating a large fungating mass at the ampulla. b ERCP demonstrating extrahepatic bile duct dilatation with tapering to the level of the ampullary tumour. c Insertion of a distal CBD metal stent with free flow of contrast into the duodenum.

The patient had an unremarkable admission after distal CBD metal stent insertion and was discharged without complications. His bilirubin normalised on discharge with corresponding improvement of other liver function tests. The increase in the duodenal mass was deemed most likely due to disease flare at the commencement of immunotherapy, so he was continued on pembrolizumab. At the time of submission, he has completed cranial radiotherapy and 36 weeks of immunotherapy with progression imaging demonstrating an ongoing treatment response.

\section{Discussion}

This case demonstrates the incidental finding of duodenal metastasis of NSCLC on FDG PET/CT and subsequent biopsy, which subsequently resulted in the patient presenting with biliary obstruction shortly after the diagnosis was made, requiring ERCP and biliary stent insertion.

Lung cancer is the leading cause of cancer death worldwide, with an estimated 1.6 million deaths each year, and approximately $50 \%$ of these patients have metastatic disease at the time of diagnosis $[1,5]$. Of those diagnoses, approximately $85 \%$ have a group of histological subtypes known as NSCLC, of which lung adenocarcinoma and lung squamous cell carcinoma are the most common types [6]. NSCLC has a predominance for distal metastasis, with bone, brain, adrenal glands, and the kidneys being the most common sites, with the occurrence of metastasis to the gastrointestinal tract from lung cancer being a rare but well-recognised phenomenon [1, 7-9]. The incidence of gastrointestinal metastasis of primary lung malignancy on autopsy studies has been published to be as high at $14 \%$; however, the actual incidence of lung cancer metastasising to the gastrointestinal tract is uncertain with approximations ranging from 0.5 to $10 \%$ depending on the method of evaluation (endoscopy, surgical specimen, or autopsy) $[7,10]$. Although adenocarcinoma has been previously demonstrated to be the most frequent histological subtype of NSCLC to metastasise to the gastrointestinal tract, there is evidence to suggest otherwise $[1,7,8,11]$.

Metastatic disease to the gastrointestinal tract by lung cancer is deemed to be a sign of late-stage disease, namely due to the diffuse spread of the tumour via the haematogenous route, and is usually detected in those patients with a previous history of lung malignancy. In contrast to this, the detection of primary lung malignancy on presentation with symptoms attributable to gastrointestinal involvement is a rare phenomenon [11].

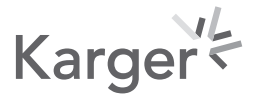


ERCP-guided biliary drainage is deemed the current standard of treatment for malignant biliary obstruction when surgery is not an option, with a success rate approaching $95 \%$ and a low risk of adverse events [12-14]. In patients in which peritonitis or obstruction is suspected with concomitant biliary metastasis, a palliative surgical approach is recommended for symptom relief [15].

\section{Conclusion}

Although uncommon, this case indicates that for lesions identified in the duodenum with high FDG avidity, metastasis should be considered. In addition to this, in a patient with known duodenal metastasis, clinicians should be aware of the potential for biliary obstruction and obstructive pancreatitis as seen in this case, and of the potential for rapid disease progression as demonstrated in the previous literature.

\section{Acknowledgements}

Not applicable.

\section{Statement of Ethics}

The authors declare that ethical approval was not required for this case report. Written informed consent was obtained from the patient for the publication of this case and accompanying images. A copy of the written consent is available for review by the Editor-in-Chief of this journal.

\section{Conflict of Interest Statement}

The authors disclose no conflicts of interest associated with the production of the submitted manuscript.

\section{Author Contributions}

R.S.O. and T.D. were responsible for the data collection and writing of the manuscript. W.D. was responsible for the primary care of the patient and editing of the manuscript. C.R. and D.B. were involved with the primary care of the patient, drafting and editing of the manuscript.

\section{Funding Sources}

This research did not receive any specific grant from funding agencies in the public, commercial, or not-for-profit sectors. 


\section{Case Reports in Oncology}

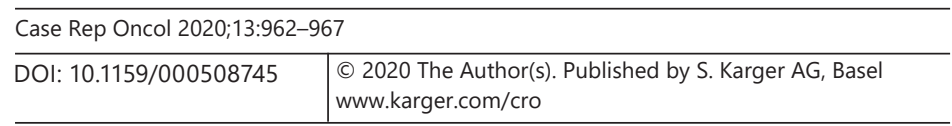

O'Neill et al.: Metastatic NSCLC Treated with Common Bile Duct Stenting

\section{References}

1 McNeill PM, Wagman LD, Neifeld JP. Small bowel metastases from primary carcinoma of the lung. Cancer. 1987;59(8):1486-9.

2 Misra S, Dwivedi M, Misra V, Dharmani S, Gupta M. Duodenal metastases from squamous cell carcinoma of the lung: endoscopic management of bleeding and biliary and duodenal obstruction. Indian J Gastroenterol. 2004; 23(5):185-6.

3 Yamada H, Akahane T, Horiuchi A, Shimada R, Shibuya H, Hayama T, et al. A case of lung squamous cell carcinoma with metastases to the duodenum and small intestine. Int Surg. 2011;96(2):176-81.

4 Hirai S, Hamanaka Y, Mitsui N, Sato K, Chatani N. Solitary metachnonous jejunum and duodenum metastasis after surgical resection of lung cancer. Kyobu Geka. 2010;63(2):129-32.

5 Torre LA, Bray F, Siegel RL, Ferlay J, Lortet-Tieulent J, Jemal A. Global cancer statistics, 2012. CA Cancer J Clin. 2015;65(2):87-108.

6 Molina JR, Yang P, Cassivi SD, Schild SE, Adjei AA. Non-small cell lung cancer: epidemiology, risk factors, treatment, and survivorship. Mayo Clin Proc. 2008;83(5):584-94.

7 Antler AS, Ough Y, Pitchumoni C, Davidian M, Thelmo W. Gastrointestinal metastases from malignant tumors of the lung. Cancer. 1982;49(1):170-2.

8 Berger A, Cellier C, Daniel C, Kron C, Riquet M, Barbier J-P, et al. Small bowel metastases from primary carcinoma of the lung: clinical findings and outcome. Am J Gastroenterol. 1999;94(7):1884.

9 Linsen PV, Linsen VM, Buunk G, Arnold DE, Aerts JG. Iron deficiency anemia as initial presentation of a nonsmall cell lung carcinoma: A case report. Respir Med Case Rep 2015;16:109-11.

10 Yoshimoto A, Kasahara K, Kawashima A. Gastrointestinal metastases from primary lung cancer. Eur J Cancer. 2006;42(18):3157-60.

11 Hillenbrand A, Sträter J, Henne-Bruns D. Frequency, symptoms and outcome of intestinal metastases of bronchopulmonary cancer. Case report and review of the literature. Int Semin Surg Oncol. 2005;2:13.

12 Castano R, Lopes TL, Alvarez O, Calvo V, Luz LP, Artifon EL. Nitinol biliary stent versus surgery for palliation of distal malignant biliary obstruction. Surg Endosc. 2010;24(9):2092-8.

13 Dhir V, Itoi T, Khashab MA, Park DH, Teoh AYB, Attam R, et al. Multicenter comparative evaluation of endoscopic placement of expandable metal stents for malignant distal common bile duct obstruction by ERCP or EUS-guided approach. Gastrointest Endosc. 2015;81(4):913-23.

14 Okamoto T, Fujioka S, Yanagisawa S, Yanaga K, Kakutani H, Tajiri H, et al. Placement of a metallic stent across the main duodenal papilla may predispose to cholangitis. Gastrointest Endosc. 2006;63(6):792-6.

15 Lee P-C, Lo C, Lin M-T, Liang J-T, Lin B-R. Role of surgical intervention in managing gastrointestinal metastases from lung cancer. World J Gastroenterol. 2011;17(38):4314. 